\title{
Acute monoarthritis associated with lipid liquid crystals
}

\author{
ANTONIO J REGINATO, H RALPH SCHUMACHER, DAVID A ALLAN, \\ AND JOSEPH L RABINOWITZ
}

From the University of Pennsylvania School of Medicine; the Veterans Administration Medical Center, Philadelphia; and Rutgers School of Medicine and Dentistry, Camden, New Jersey, USA

SUMMARY We describe three women with unexplained acute monoarthritis and birefringent lipid spherules or lipid liquid crystals in their synovial fluid. Synovial fluid aspirated within 24 hours of onset showed leucocyte counts ranging from $10 \times 10^{9}$ to $46 \times 10^{9} / 1$ with $91-95 \%$ polymorphonuclear cells. Numerous positively birefringent $2-6 \mu \mathrm{m}$ microspherules were seen inside and outside polymorphonuclear cells. These were dissolved in alcohol-ether but were not digested by uricase. Other non-birefringent globules of similar size that stained with Sudan black B were also seen. Transmission electron microscopy identified osmiophilic homogeneous or multilamellated material in phagocytic vacuoles. Serum lipids, lipase, and repeated joint radiographs were normal. Arthritis subsided completely after treatment with colchicine in one patient and nonsteroidal anti-inflammatory agents in two. Thus birefringent microspherules can be found in association with acute, otherwise unexplained, arthritis. It seems possible that certain lipid liquid crystals may induce synovial inflammation similar to inflammation seen in other crystal induced arthritides.

Key words: synovial fluid, liposomes, articular inflammation.

Birefringent lipid microspherules, appearing as Maltese crosses, have occasionally been observed during synovial fluid analysis. ${ }^{1}$ These microspherules are formed by multilayers of phospholipids and water and are variously described as liposomes, smetic mesophases, or lipid liquid crystals. ${ }^{2}$ Recently such microspherules have been reported in the synovial fluid of one patient with otherwise unexplained acute monoarthritis ${ }^{3}$ and in the synovial fluid and synovial lining cells of another patient with chronic unexplained arthritis. ${ }^{4}$

In this report we describe three patients with acute inflammatory monoarthritis whose synovial fluid was characterised by the presence of abundant intracellular and extracellular strongly birefringent lipid liquid crystals. Transmission electron microscopy identified osmiophilic homogeneous round bodies or lamellated material in phagocytic vacuoles. In addition the lipid composition of the

Accepted for publication 7 January 1985.

Correspondence to Dr Antonio J Reginato. Division of Rheumatology, Department of Medicine. Cooper Hospital/University Medical Center, One Cooper Plaza, Camden. New Jersey 08103. USA. synovial fluid supernatant in two patients showed an increase in free fatty acids, lysolecithin and phosphatidic acid. All three patients were seen during the first 24 hours of the attack and had normal serum lipid profiles, serum amylase, lipase, and radiographs of the affected joints. In each case the arthritis subsided completely in five to 10 days after treatment with colchicine or non-steroidal antiinflammatory agents.

\section{Patients and methods}

PATIENT 1

The first patient was a 41-year-old black woman who had sarcoidosis of her lungs proved by biopsy, lymph nodes, and skin and bones which required steroid therapy for bone pain and fever. She presented with acute swelling of her left wrist. Over the previous 15 years she had experienced recurrent episodes of similar synovitis of both wrists, which had been rapidly controlled with either oral or intravenous colchicine.

$1 \mathrm{ml}$ of cloudy synovial fluid was aspirated from her acutely inflamed left wrist (Table 1). The fluid 
Table 1 Synovial fluid analyses in three patients with acute arthritis and lipid liquid crystals

\begin{tabular}{|c|c|c|c|c|c|c|c|}
\hline & Patient No. I & Patient No. & 2 & & Patient No. & 3 & \\
\hline Days & 1 & 2 & 3 & 10 & 1 & 6 & 8 \\
\hline Appearance & Cloudy & Bloody & Cloudy & Clear & Cloudy & Bloody & Bloody \\
\hline Amount (ml) & 1 & 40 & 20 & 10 & 50 & 5 & 3 \\
\hline Viscosity & Poor & Poor & Poor & Poor & Poor & Poor & Poor \\
\hline Leucocytes/l & $10 \times 10^{9}$ & $10.4 \times 10^{4}$ & $24.4 \times 10^{4}$ & $0.85 \times 10^{4}$ & $40.5 \times 10^{4}$ & $27 \times 10^{4}$ & $12 \times 10^{4}$ \\
\hline Polymorphonuclear cells ( $\%$ ) & 75 & 94 & 92 & 40 & 95 & 46 & 28 \\
\hline $\begin{array}{l}\text { Lipid crystals } \\
\text { Bacterial culture }\end{array}$ & $\begin{array}{l}+++ \\
\mathrm{NG}^{*}\end{array}$ & $\begin{array}{l}+++ \\
N G\end{array}$ & $\begin{array}{l}++ \\
\text { NG }\end{array}$ & $\begin{array}{l}+ \\
\text { NG }\end{array}$ & $\begin{array}{l}+t+ \\
\mathrm{NG}\end{array}$ & $\begin{array}{l}++ \\
\mathrm{NG}\end{array}$ & $\stackrel{+}{N G}$ \\
\hline
\end{tabular}

showed decreased viscosity with a leucocyte count of $10 \times 10^{9}$ cells/l with $75 \%$ polymorphonuclear neutrophils. A fresh drop preparation showed abundant strongly birefringent intracellular and extracellular microspherules (Fig. 1). No other crystals were seen. Other laboratory studies including serum lipid profile, serum lipase and amylase, calcium, phos-

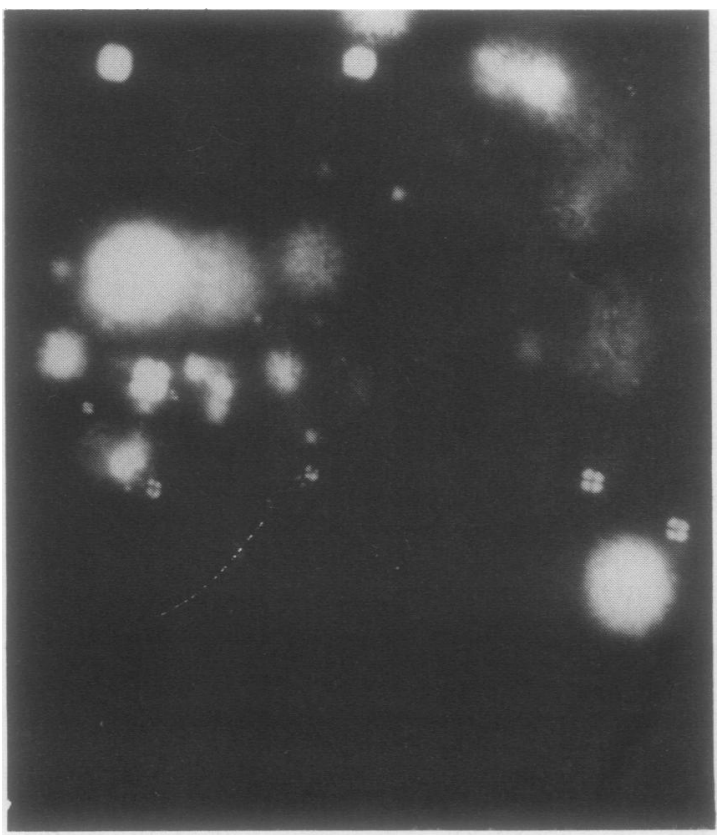

Fig. 1 a

Figure 1 Synovial fluid, fresh preparation of patient No. 1. (a) Abundant intracellular and extracellular doubly birefringent microspherules. Regular polarised light. $(\times 42)$. (b) Strongly positively birefringent microspherules and $a$ single larger spherule (arrow). The large line indicates the axis of slow vibration of the compensator. The dark portions of the spherule are parallel to the marked axis. Compensated polarised light. $(\times 60)$. (c) A single large spherule. Compensated polarised light. $(\times 200)$. phorus, alkaline phosphatase, and uric acid were within normal limits. Repeated radiographs and ${ }^{99 \mathrm{~m}} \mathrm{Tc}$ pyrophosphate bone scan of her hands after the inflammation subsided were unremarkable. Sheo received oral colchicine $0.6 \mathrm{mg}$ four times a day, and her left wrist synovitis subsided completely withim five days.

PATIENT 2

A 21-year-old black woman came to the emergencyoo room complaining of a painful swollen left knee of

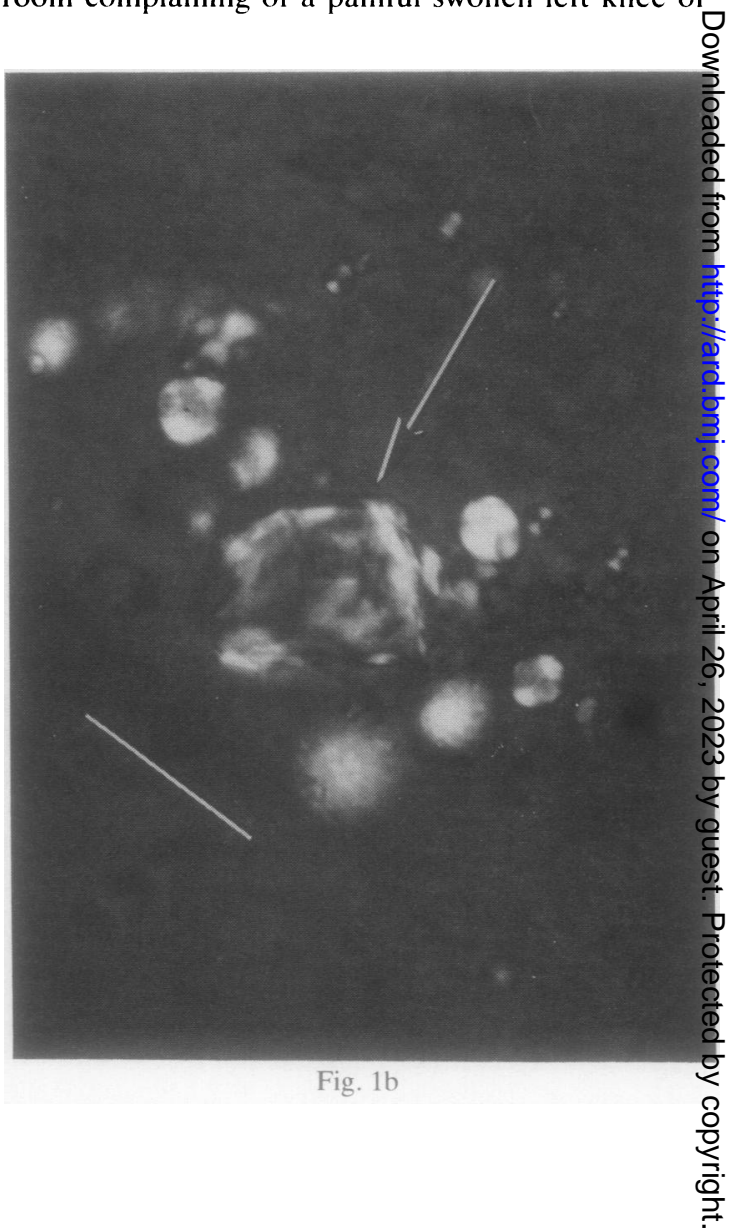




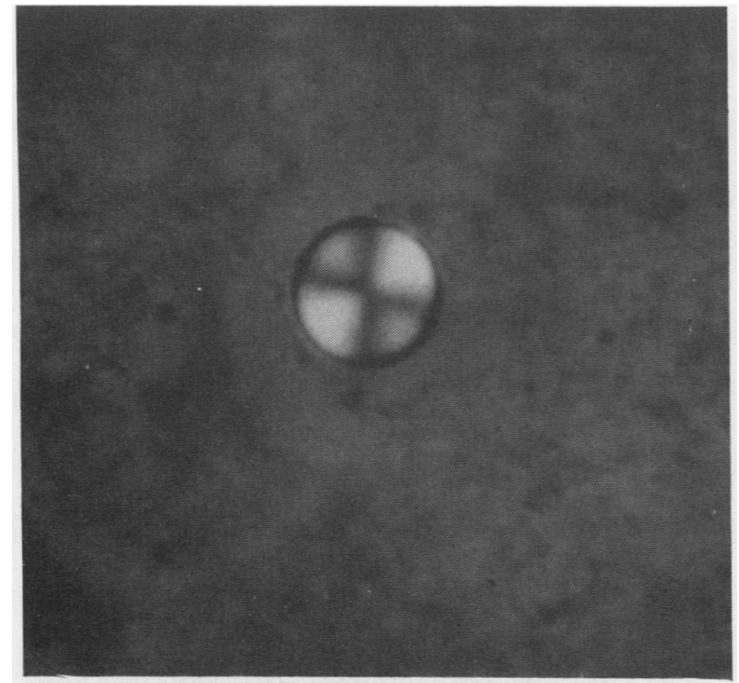

Fig. 1c

24 hours' duration. Three weeks before she had fallen to the floor landing on her right knee but had experienced no pain in either knee subsequent to the fall. Her left knee appeared swollen, warm, and tender with flexion limited to $50^{\circ}$. Synovial fluid was aspirated from her left knee on the second, third, and tenth days of her arthritis (Table 1). The fluid which was initially haemorrhagic became cloudy by the third day with decreased viscosity and leucocyte counts up to $24.4 \times 10^{9}$ cells $/ 1$ with $92 \%$ polymorphonuclear cells. Abundant lipid liquid crystals similar to those observed in the first patient were seen inside and outside of the polymorphonuclear neutrophils. No other crystals were seen. By the 10th day the synovial fluid aspirate was clear and had a total cell count of $0.85 \times 10^{9}$ cells $/ 1$ with $40 \%$ polymorphonuclear cells and few lipid liquid crystals. Bacterial cultures of blood and synovial fluid were negative. Serum lipid profile, amylase, lipase, uric acid, and ${ }^{99 m}$ Tc pyrophosphate bone scan were all unremarkable. Left knee synovitis subsided completely after 14 days of treatment with $75 \mathrm{mg}$ of indomethacin daily.

PATIENT 3

A 54-year-old white woman with Laennec's cirrhosis proved by biopsy developed acute painful swelling of her right knee associated with fever and chills. Physical examination showed a temperature of $101.6^{\circ} \mathrm{F}$ and a warm swollen right knee with flexion limited to $60^{\circ}$. Synovial fluid was aspirated from her right knee on the first, sixth, and eighth days of her arthritis (Table 1). The fluid which was initially cloudy with decreased viscosity had a leucocyte count of $40.5 \times 10^{9}$ cells $/ 1$ with $95 \%$ polymorphonuclear cells. A fresh drop preparation showed abundant intracellular and extracellular strongly positively birefringent Maltese cross like liquid crystals. No other crystals were seen. On subsequent synovial fluid analysis on day 8 the total cell count was $12 \times 10^{y}$ cells $/ 1$ with only $28 \%$ polymorphonuclear cells and few lipid liquid crystals. Bacterial cultures of both synovial fluid samples and blood showed no bacterial growth. Repeat radiographs of her knees and serum biochemical profile were unremarkable. She was treated with antibiotics for two days followed by indomethacin $100 \mathrm{mg}$ daily, which resulted in complete resolution of her knee synovitis by the 10 th day.

\section{SYNOVIAL FLUID STUDIES}

\section{Lipid staining}

Synovial fluid smears of all three patients were stained with Sudan black B stain for lipids. ${ }^{5} 1 \mathrm{ml}$ synovial fluid aliquots from patients 2 and 3 were mixed with $1 \mathrm{ml}$ of $1: 1$ alcohol:ether at room temperature and observed under compensated polarised light.

\section{Uricase digestion}

Uricase digestion of the microspherules was attempted with a single drop of uricase solution of 75 units of activity per $2 \mathrm{ml}$ of distilled water on a glass slide with equal amounts of synovial fluid. The slide was sealed with a coverslip, and individual lipid liquid crystals were observed during the next 24 hours. $^{6}$

\section{Electron microscopic studies}

Synovial fluid pellets from patients 2 and 3 were fixed in half strength Karnovsky's glutaraldehydeparaformaldehyde fixative. After 15 minutes fixation the pellets were minced into $1 \times 1 \mathrm{~mm}$ pieces, fixed for four more hours at room temperature, and processed by a previously reported technique. ${ }^{7}$ Specimens were examined in a Zeiss EM10 transmission electron microscope with a $60 \mathrm{kV}$ beam.

\section{Biochemical lipid analysis}

Synovial fluids of patients 2 and 3 were available for biochemical lipid analysis. Fluid was centrifuged for 3-5 minutes at $100 \mathrm{~g}$. Total lipids, neutral lipids and phospholipids were measured in accordance with a technique previously reported by the authors in a study of lipid composition of the tissue of normal human knee joints. ${ }^{8}$ Values for total lipids, neutral lipids, and phospholipids from normal pooled synovial fluid from that study were used as controls. ${ }^{8}$ 


\section{Results}

LIPID STAINING, ALCOHOL:ETHER SOLUBILITY AND URICASE DIGESTION All three synovial fluid specimens showed Sudan black B stained fat droplets. Compensated polarised light showed abundant 2-6 $\mu \mathrm{m}$ birefringent microspherules with a Maltese cross appearance. The strongly positive birefringent microspherules were completely dissolved in a mixture of $1: 1$ alcohol:ether but were resistant to uricase digestion.

\section{TRANSMISSION ELECTRON MICROSCOPIC} STUDIES

Under transmission electron microscopy two predominant types of pertinent intracellular inclusions were observed. Some phagocytic vacuoles contained homogeneous grey lipid droplets (Fig. 2a). Additional homogeneous grey lipid inclusions similar to those described by Zucker-Franklin ${ }^{9}$ were seen in the cytoplasm (Fig. 2b). Other vacuoles had multilayered lamellated inclusions similar to the arrangement reported by others in lipid liquid crystals. ${ }^{2}$ Some of these inclusions showed a dark dense

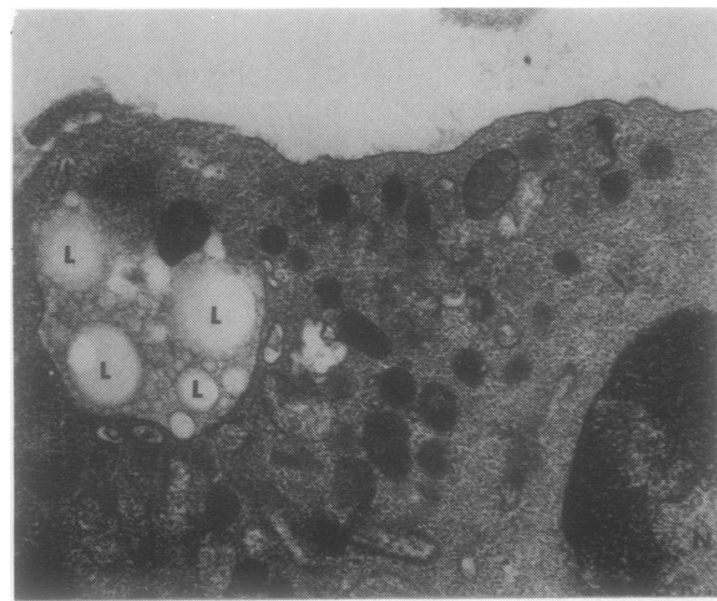

\section{Fig. 2a}

Fig. 2 Electron micrographs of synovial fluid cells with intracellular lipid inclusions. (a) Homogeneous pale lipid droplets $(L)$ in a vacuole of a polymorphonuclear leucocyte. $N=$ nucleus. Patient $2 .(\times 20$ 000). (b) Single grey lipid $(L)$ inclusions in a mononuclear cell. $N=$ nucleus. Patient 2.

(×30 200). (c) Large multilamellated inclusion (MLI) with a dense centre $(D)$ surrounded by smaller lamellated inclusions (arrows) and an isolated denser body (D) all in a synovial cell vacuole. Patient $3 .(\times 28700)$. (d)

Homogeneous lipid droplets $(L)$ with membranous arrays at their margins (arrows) and multilamellated arrays in vacuoles of synovial mononuclear cell. Patient 3. $(\times 18150)$.

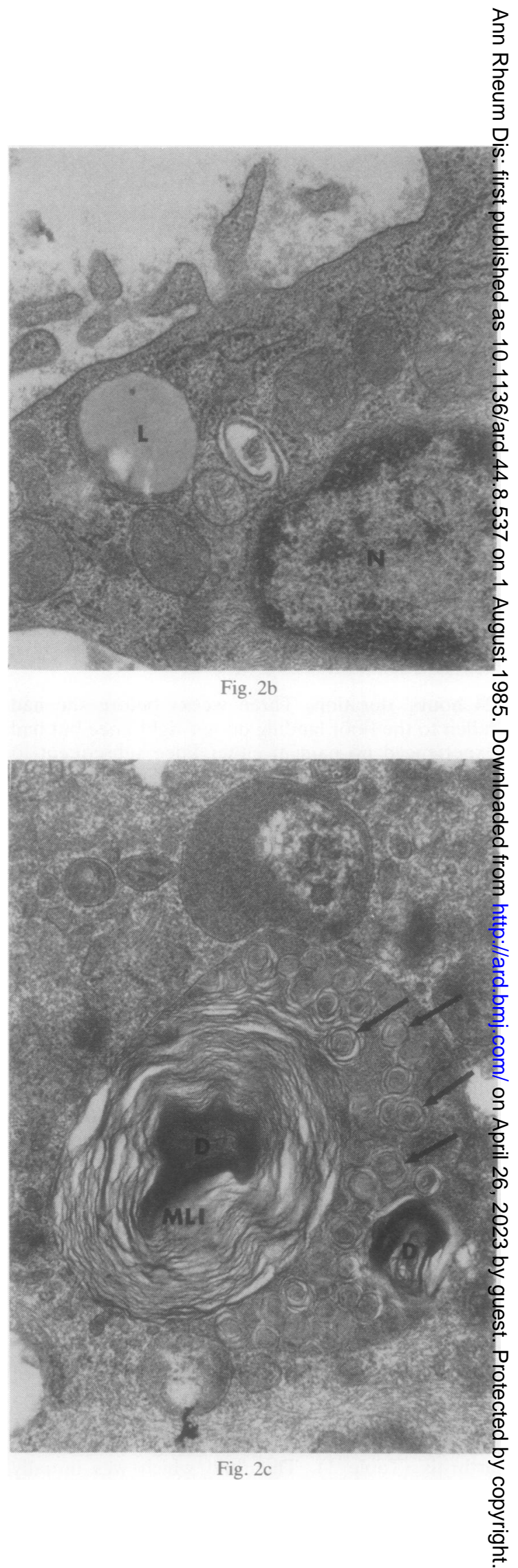




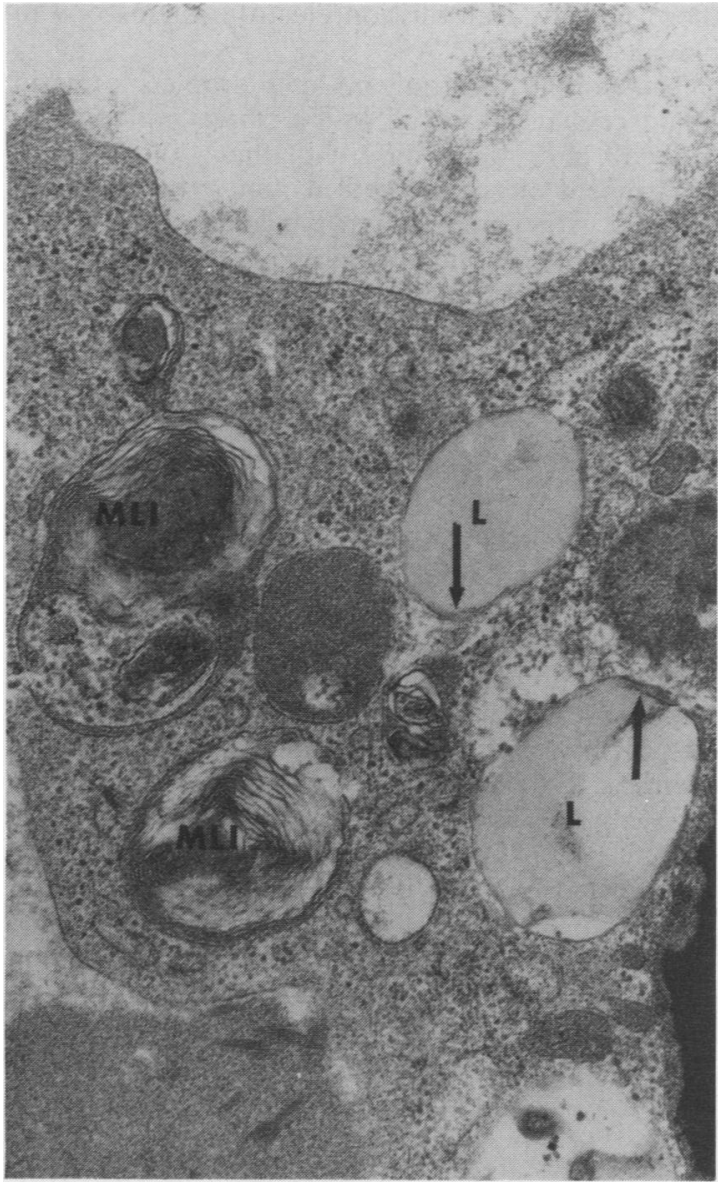

Fig. 2d centre and were surrounded by numerous smaller but identical lamellated inclusions (Fig. 2c). In some areas a suggestion of transition from homogeneous to membranous inclusions was seen, with small membranous arrays at the margin of a homogeneous deposit (Fig. 2d). These inclusions resembled particles which have been described in foamy mouse macrophages in tissue culture. ${ }^{10}$ No viral particles or solid crystals were observed in either of the two synovial fluids studied.

\section{BIOCHEMICAL LIPID STUDIES}

Table 2 compares the synovial fluid concentrations of total lipids, neutral lipids, and phospholipids of patients 2 and 3 with normal synovial fluid pooled from cadavers from a previous study using a similar analysis. ${ }^{8}$ There was a slight but insignificant increase in the percentage of total lipids of $9.8 \mathrm{mg}$ and $8.8 \mathrm{mg}$ compared with $5 \mathrm{mg}$ in the normal fluid. Increased concentrations of fatty acids, lysolecithin and phosphatidic acid and a decrease in lecithin concentration were observed in our patients compared with normal controls.

\section{Discussion}

LIPID LIQUID CRYSTAL CONCEPT

We describe three patients who developed an acute monoarthritis in association with the presence in the synovial fluid of positively birefringent microspherules which had features of lipid liquid crystals under compensated polarised light.

Liquid crystals represent states of matter having characteristics of both liquids and crystalline solids. ${ }^{2} 1112$ They have some order in their molecular arrangement and also some degree of fluidity. Those molecules which tend to form liquid crystals

Table 2 Lipids in the synovial fluid of two patients with acute monoarthritis associated with lipid crystals

\begin{tabular}{lccc}
\hline & Patient No. 2 & Patient No. 3 & Normal pooled synovial fluid \\
\hline Total lipids (mg \%) & $9 \cdot 8$ & $8 \cdot 8$ & $5 \pm 0 \cdot 8^{*}$ \\
Neutral lipids (\%) & & & \\
Triglycerides (\%) & $75 \cdot 5$ & $72 \cdot 7$ & $65 \cdot 5 \pm 5 \cdot 1$ \\
Mono-, diglycerides (\%) & $19 \cdot 0$ & $12 \cdot 7$ & $15 \cdot 7 \pm 2 \cdot 1$ \\
Free fatty acids (\%) & $2 \cdot 8$ & $2 \cdot 8$ & $2 \cdot 5 \pm 1$ \\
Free and esterified cholesterols (\%) & $8 \cdot 9$ & $8 \cdot 7$ & $1 \cdot 7 \pm 0 \cdot 7 \mathrm{p}<0 \cdot 001$ \\
& $43 \cdot 5$ & $47 \cdot 5$ & $3 \cdot 1 \pm 5 \cdot 3$ \\
Total phospholipids (\%) & $24 \cdot 5$ & $26 \cdot 3$ & $32 \cdot 5 \pm 3 \cdot 7$ \\
Lecithin (\%) & $1 \cdot 4$ & $1 \cdot 7$ & $14 \cdot 8 \pm 3 \cdot 7 \mathrm{p}<0 \cdot 001$ \\
Cephalin (\%) & $3 \cdot 7$ & $4 \cdot 8$ & $6 \cdot 7 \pm 1 \cdot 8$ \\
Phosphatidyl serine (\%) & $2 \cdot 8$ & $3 \cdot 5$ & $4 \cdot 3 \pm 2 \cdot 5$ \\
Lysolecithin (\%) & $1 \cdot 4$ & $1 \cdot 7$ & $0 \cdot 6 \pm 0 \cdot 2 \mathrm{p}<0 \cdot 01$ \\
Phosphatidic acid (\%) & $2 \cdot 8$ & $3 \cdot 1$ & $0 \cdot 9 \pm 0 \cdot 4 \mathrm{p}<0 \cdot 01$ \\
Sphingomyelin (\%) & $3 \cdot 5$ & $4 \cdot 5$ & $4 \cdot 5 \pm 1$ \\
\hline
\end{tabular}

*Values are mean \pm SD. 
are longer than they are wide and have a polar or aromatic part on the molecule. The shape and polar interaction permit the molecules to align in partially ordered arrays described as 'smetic' or layered arrays. This is similar to the arrangement seen in liposomes and is in contrast to nematic arrays, in which the molecules are aligned side by side but not in mandatory layers. ${ }^{11} 12$ Liquid crystals can be formed in vitro in two known ways: $(a)$ molecules when heated may not melt directly to isotropic liquid but may instead pass through an intermediate state of mesophase or liquid crystal (thermotropic liquid crystal); (b) molecules can be partially liquefied by the action of a solvent producing lyotropic liquid crystals. Lyotropic crystals can also undergo additional thermotropic transition. ${ }^{2}$

A liquid crystalline order of molecules has been described in healthy and pathological biological systems, such as in normal cell organelles, myelin bile, and lipoproteins; and in arteriosclerotic plaques, and lipid storage diseases. ${ }^{11}$

LIPID LIQUID CRYSTALS AND ARTHRITIS It is not unusual to find cholesterol crystals and birefringent lipid microspherules in chronically inflamed joints and bursae of patients with rheumatoid arthritis. ${ }^{13}$ They are usually associated with increased synovial fluid lipid concentration and probably are a result of marked cell membrane damage and phospholipid release. The three patients in this report and the one described by Weinstein $^{3}$ differ in that each patient developed acute monoarthritis which was associated with synovial fluid lipid liquid crystals. All these four patients experienced acute onset of monoarticular synovitis associated with florid signs of acute inflammation, synovial fluid leucocytosis, and abundant extracellular and/or intracellular positively birefringent microspherules. Arthritis subsided in all patients within a week after the administration of non-steroidal anti-inflammatory agents. The clinical presentation and course are similar to those seen in patients with acute gout or other solid crystal induced arthritis and therefore introduces the possibility that lipid liquid crystals might induce inflammation as has been proposed for solid crystals. The decrease in numbers of liquid crystal as the attacks subside supports their role as an aetiologic factor producing inflammation.

However, we cannot completely exclude the alternative possibility that these liquid crystals are only a result of acute injury of the cell membrane by an inflammatory process induced by some other as yet unidentified phlogistic agent. Partially digested platelets which are rich in phospholipids have been reported to show multilamellated structures like those seen by transmission electron microscopy in ? patients 2 and $3 .^{14}$

The double birefringent lipid droplets or lipid liquid crystals observed in the three patients described here are different than those non- $\frac{\bar{c}}{\bar{c}}$ birefringent ones observed in joint effusions from? patients with joint trauma, pancreatic induced fato necrosis, and aseptic bone necrosis. In these condi-ñ tions non-birefringent neutral fat droplets can $\vec{b}$ escape from the underlying fatty synovium or bonemarrow. ${ }^{815}$

\section{ELECTRON MICROSCOPIC STUDIES}

Most of the lipid deposits observed in patients 2 andis 3 displayed arrays of concentric layers similar too those described in lipid liquid crystals andi cholesteryl ester lipid droplets. ${ }^{11}{ }^{11}$ Some of these multilamellated inclusions (Fig. 2c) were also similar to those myelin-like figures observed in the tissue of patients with Fabry's disease. ${ }^{16}$

Other less numerous homogeneous grey droplet like inclusions were also seen (Fig. 2b) and were? identical to the type described by Schlesinger et al: $\overrightarrow{0}$ in synovial fluid macrophages of patients withor chronic polyarthritis. ${ }^{4}$ The finding of these two typesof inclusion bodies is in agreement with the studies of McGookey and Anderson who have described the formation of cholesteryl ester lipid droplets in tissue cultured mouse macrophages. ${ }^{10}$ They havep shown that cholesteryl esters are initially seques trated in homogeneous neutral lipid droplets. Sub을 sequently, focal membranous cholesterol containing arrays accumulate in the homogeneous deposits These arrays are first at the margin of the droplet when cholesterol concentration increases the drople 5 turns into a multilamellated myelin-like structure, as was seen in the two patients described here. ${ }^{10}$ This observation suggests that at least some local in tracellular formation of inclusions is possible. The appearance of some droplets and arrays in memo brane bounded vacuoles (Figs $2 \mathrm{a}$ and $2 \mathrm{c}$ ) implies that the liquid crystals can also be phagocytisedo

LIPID LIQUID CRYSTALS AND

I N F L A M M A T I O N

At the present time there is no direct evidence than lipid liquid crystals can induce inflammation in joints or elsewhere. Impure preparations of lipids or fatty acids have produced mild inflammation afte? intra-articular injection. Low density lipoproteins can be shown to be cytotoxic to fibroblasts in tissue cultures. ${ }^{18}$ Lipid liquid crystals or liposomes haves been used as carriers of synthetic corticosteroids fo $\vec{D}$ intra-articular injections but to date no postinjection inflammation has been reported. ${ }^{19}$ It seems possible that the ability of these microspherules to induce 
inflammation might vary with the nature of the phospholipids contained in the liquid crystals.

In a study of gout negatively birefringent urate spherulites, different from solid crystals and possibly analogous to liquid crystals, have been described in one synovial fluid with acute arthritis as the only form of urate. ${ }^{6}$ We have observed them in another patient in association with typical strongly negatively birefringent monosodium urate crystals. These spherulites can also be observed during synthetic preparation of monosodium urate crystals at a lower temperature. ${ }^{6}$ Uric acid and calcium phosphate crystalline spherulites like those discussed here are part of the normal physiology of kidney and gastrointestinal tract in birds and insects (unpublished data).

As with gout and calcium pyrophosphate deposition disease levels of the soluble materials in the synovial fluid were not useful in the evaluation of our patients. The increases in some lipids in our patients compared with normal pooled synovial fluid will need to be compared further with other inflammatory fluids.

Because the mechanisms of many musculoskeletal manifestations of several types of hyperlipidaemias, such as polyarthralgias, episodic arthritis, and fibrositis, are poorly understood, the possible role of lipid liquid crystals like those reported here deserves to be investigated. Arthralgia and arthritis which is exacerbated by warm weather have been observed in patients with Fabry's disease. ${ }^{20}$ Synovium or other tissues examined in these patients with Fabry's disease have shown glycolipidic inclusions with features of lipid liquid crystals. ${ }^{16} 20$

Whether the sarcoidosis and cirrhosis seen in patients 1 and 3 could be related in any way to the lipid liquid crystals can be only speculation at this time. The minor trauma in patient 2 of course can be considered as a possible factor in release of lipids into joint fluid.

Confirmation of the possible phlogistic role of the liquid crystals will require in-vitro and in-vivo experiments similar to those previously conducted with the solid crystals of urate, apatite, and calcium pyrophosphate. However, because the ability of these molecules to display a crystalline arrangement is temperature dependent, their synthesis and handling will require more sophisticated techniques than those used in the study of solid crystals in joint fluid. ${ }^{21}$
The authors acknowledge the superb technical assistance of Gilda Clayburne, Susan Rothfuss, and Marie Sieck and the secretarial assistance of Mary Ellen McGuire and Anita Boyd.

\section{References}

1 Wild J H, Zvaifler $\mathrm{N} \mathrm{J}$. An office technique for identifying crystals in synovial fluid. Am Fam Physician 1975; 12: 72-81.

2 Banghan A D. Development of the liposome concept. In. Gregoriadis G, Allison A C, eds. Liposomes in biological systems. New York: Wiley, 1980: 7-19.

3 Weinstein J. Synovial fluid leukocytosis associated with intracellular lipid inclusions. Arch Intern Med 1980; 140: 560-1.

4 Schlesinger P A, Stillman M T, Peterson L. Polyarthritis with birefringent lipid within synovial fluid macrophages: case report and ultrastructural study. Arthritis Rheum 1982; 25: 1365-8.

5 Pearse A G E. Histochemistry, theoretical and applied. Edinburgh: Churchill Livingstone, 1972.

6 Fiechtner J J, Simkin P A. Urate spherulites in gouty synovia. JAMA 1981; 247: 1533-6.

7 Reginato A J, Schumacher H R, Brighton C T. Experimental hydroxyapatite synovial and articular cartilage calcification: light and electron microscopic studies. Arthritis Rheum 1982; 25: $1239-49$.

8 Rabinowitz J D, Gregg J R, Nixon J E. Schumacher H R. Lipid composition of the tissues of human knee joints. 1. Observations in normal joints (articular cartilage, meniscus, ligaments, synovial fluid, synovium, intra-articular fat pad and bone marrow). Clin Orthop 1979; 143: 260-5.

9 Lutas M, Zucker-Franklin D. Formation of lipid inclusions in normal blood leukocytes. Blood 1977: 49: 309-10.

10 McGookey D J, Anderson R G W. Morphological characterization of the cholesteryl ester cycle in cultured mouse macrophage foam cells. J Cell Biol 1983; 97: 1156-68.

11 Small D M. Liquid crystals in living and dying systems. $J$ Colloid Interface Sci 1977; 58: 581-602.

12 Ferguson J L. Liquid crystals. Sci Am 1964; 211: 77-85.

13 Taccari E, Teodori S, Manelli H. Les épanchements chyliformes de la polyartrite chronique évolutive. Études cytochimiques et ultrastructurales des cellules spumeuses. Rev Rhum Mal Osteoartic 1982; 48: 263-71.

14 Lasser A. Diffuse histiocytosis of the spleen and idiopathic thrombocytopenic purpura histochemical and ultrastructural studies. J Clin Pathol 1983; 80: 529-33.

15 Currey H L F, Vernon, Roberts B. Examination of synovial fluid. Clin Rheum Dis 1976; 2: 149-77.

16 Nussbaum A I. Laborer presents unexplained progressive arthralgia. Workshops in rheumatology 1982; 3: 1-3.

17 Weinberger A, Schumacher H R. Experimental joint trauma: synovial response to blunt trauma and inflammatory reaction to intraarticular injection of fat. $J$ Rheumatol 1981; 8: 380-9.

18 Morel D W, Hessler J R, Chisolm G M. Low density lipoprotein cytotoxicity induced by free radical peroxidation of lipid. J Lipid Res 1983; 24: 1070-6.

19 Shaw I H, Dingle J T. Liposomes as steroid carriers in the intraarticular therapy of rheumatoid arthritis. In: Gregoriadis $\mathrm{C}$, Allison AC, eds. Liposomes in biological systems. New York: Wiley, 1979: 299-323.

20 Sheith K J, Bernhard G C. The arthropathy of Fabry's disease. Arthritis Rheum 1979; 22: 281-3.

21 Small D M. The physical state of biological importance: cholesteryl esters, cholesterol, triglyceride. In Blank $\mathbf{M}$, ed. Surface chemistry of biological systems. New York: Contact Associated International Ltd, 1979: 55-64. 